\title{
Combined Intense Pulsed Light and Low-Level Light Therapy for the Treatment of Dry Eye: A Retrospective Before-After Study with One- Year Follow-Up
}

Miguel Angel Pérez-

Silguero'

David Pérez-Silguero $\mathbb{D}^{2}$

Amado Rivero-Santana $\left(\mathbb{D}^{3}\right.$

Maria Inmaculada Bernal-

Blasco $^{4}$

Pablo Encinas-Pisa'

'Department of Ophthalmology, La Paloma Hospital, Las Palmas de Gran Canaria, Canary Islands, Spain;

${ }^{2}$ Department of Ophthalmology, PérezSilguero Ophthalmologic Clinic, Las Palmas de Gran Canaria, Canary Islands, Spain; ${ }^{3}$ Department of Health Technology Assessment, Fundación Canaria Instituto de Investigación Sanitaria de Canarias (FIISC), Las Palmas de Gran Canaria, Canary Islands, Spain; ${ }^{4}$ Department of Community and Family Medicine, Primary Care Center of Cuevas Torres, Las Palmas de Gran Canaria, Canary Islands, Spain
Correspondence: Amado Rivero-Santana Servicio de Evaluación del Servicio Canario de la Salud, Camino Candelaria, 44, El Rosario, S/C de Tenerife, 38I09, Spain

Tel +34 922478269

Email amado.riverosantana@sescs.es
Purpose: To assess the effectiveness of a combination of intense pulsed light and low-level light therapy (IPL/LLLT) for the treatment of dry eye.

Study Design: Retrospective before-after single-center clinical study.

Materials and Methods: Patients diagnosed with dry eye, refractory to conventional treatment, underwent four sessions of combined IPL/LLLT over 3 months. The Ocular Surface Disease Index (OSDI) questionnaire, non-invasive breakup time (NIBUT), tear film osmolarity and meniscus height were measured 6 months before intervention, at baseline, post-intervention (3 months), 9 and 15 months.

Results: NIBUT, osmolarity and meniscus height significantly worsened during the 6 months before treatment, whereas symptoms did not change. OSDI scores significantly improved at post-intervention $(\mathrm{MD}=-44.0,95 \% \mathrm{CI}-38.1,-50.0)$, and then increased again until the at last follow-up, but still significantly different from baseline ( $\mathrm{MD}=-30.0$, 95\% CI $-23.4,-36.8$ ). The three clinical signs showed a similar pattern, with one-year improvements of 3.6 seconds for the NIBUT (95\% CI 3.1, 4.2, p <0.001), $28 \mathrm{mOsm} / \mathrm{L}$ for osmolarity (95\% CI 23.6, 32.4, p <0.001) and $0.03 \mathrm{~mm}$ for meniscus height $(95 \%$ CI 0.02 , $0.04, \mathrm{p}<0.001)$. No adverse effects were observed.

Conclusion: IPL/LLLT is safe and produces an important reduction in symptoms and signs of dry eye disease, still relevant one year after the end of treatment in a sample with high symptoms' severity. Therefore, it represents a promising treatment option for patients who do not improve with conventional treatment. Randomized trials are needed to determine the added benefit provided by LLLT.

Keywords: dry eye, meibomian gland dysfunction, intense pulsed light, low-level light therapy, retrospective study

\section{Introduction}

Dry eye disease (DED) affects millions of people worldwide. ${ }^{1}$ Its symptoms include itching, stinging, burning, irritation, foreign body sensation, photophobia or pain, among others, affecting considerably patients' quality of life in severe cases. ${ }^{2}$ One third of DED patients also show Meibomian Gland Dysfunction (MGD), a condition of the meibomian glands characterized by terminal duct obstruction and/or changes in their secretions, which is considered the leading etiologic factor of DED. ${ }^{1-3}$ 
Conventional treatment for DED follows a stepwise approach, ${ }^{4}$ starting with patient education, environmental and dietary modifications, and conservative treatments like lid hygiene, warm compresses, meibomian gland expression, and artificial tears. Medications include topical steroids, cyclosporine, leucocyte function-associated antigen-l antagonists, secretagogues, and topical or oral antibiotics. Refractory cases are treated with long-term topical corticosteroids, amniotic membrane grafting, or surgical interventions.

Intense Pulsed Light (IPL) therapy was developed and has been widely used to treat dermatologic diseases, but casual observations of its effects on Meibomian glands and symptoms of DED suggested that it could also be effective for this condition. ${ }^{5}$ This technique consists in the application of a series of pulses of non-coherent polychromatic light in the periorbital region, with a wavelength spectrum ranging $500-1200 \mathrm{~nm}$. This produces a selective thermal effect on the irradiated tissue, leading to coagulation and ablation of blood vessels and thus reducing vascularization. ${ }^{6}$ Although its mechanisms of therapeutic action are still being investigated, $^{7-9}$ some randomized trials and several case series have shown that IPL is safe and effective in improving MGD and reducing symptoms of DED. ${ }^{10-14}$

Another kind of photobiomodulation is the Low-Level Light Therapy (LLLT). This technique uses light-emitting diodes (LEDs) at wavelengths insufficient to produce a thermal effect (often 590-633 nm), but that increases photon intensity and its capacity to penetrate below the skin, inducing cellular photoactivation. ${ }^{15}$ In patients with DED, Toyos et al ${ }^{16}$ observed a significant increase in Tear film Breakup Time (TBUT) after 3 months of red-light treatment, and Stonecipher et $\mathrm{al}^{17}$ obtained significant improvements in symptoms, TBUT and MGD after 3 sessions over one week.

Recently, Stonecipher et al ${ }^{18}$ published a retrospective analysis of 230 patients with MGD treated in one session with a combination of IPL and LLLT. One to three months after treatment, statistically and clinically significant improvements were observed in symptoms, such as TBUT and meibomian dysfunction grade, with no facial or ocular adverse effects. The aim of our study is to add new evidence on the effectiveness of this combined light therapy in the treatment of DED.

\section{Materials and Methods}

This study is a retrospective chart analysis of patients treated with IPL/LLLT in an ophthalmologic clinic in Gran Canaria (Spain), between January 2017 and June 2018. The
Institutional Review Board of the Hospital La Paloma, Gran Canaria (ref: 2019/0037) approved the study, which was carried out in accordance with the tenets of the Declaration of Helsinki. Patients presented a diagnostic of evaporative dry eye, based on the criteria of the Tear Film and Ocular Surface Society (TFOS-DEWS II): ${ }^{19}$ a score $\geq 13$ in the Ocular Surface Disease Index (OSDI) questionnaire, ${ }^{20}$ TBUT $<10$ seconds, osmolarity $>308 \mathrm{mOsm} / 1$ or a difference between eyes higher than $8 \mathrm{mOsm} / 1$, and the presence of lipid abnormalities. They were being treated with artificial tears, antibiotics or topical steroids (none with systemic treatment), showing no improvement or frequent relapses. All patients had Fitzpatrick skin types between I-V levels, ${ }^{21}$ for whom IPL treatment is indicated. After being informed about IPL/ LLLT, they signed informed consent and underwent the first session. They continued their conventional treatment during IPL/LLLT and follow up.

We included patients in the analysis if they were adults who had not been treated for other ocular problems, nor had any incident in the eyes during the IPL/LLLT period or follow-up, and who had completed the assessment of the dependent variables 6 months before treatment, at baseline, post-treatment (3 months) and last follow-up (12 months after the end of treatment).

\section{Treatment}

Treatment was applied with the CE-Marked Eye-light ${ }^{\circledR}$ device (Espansione Marketing S.p.A., Bologna, Italy). It consisted of 4 sessions over 3 months (weeks $0,1,4$ and 12), and was applied following manufacturer's recommendations and as described in Stonecipher et al. ${ }^{18}$ Patients were sitting or in supine position, wearing the protective opaque goggles recommended by the manufacturer. In each eye, five IPL pulses (wavelength $600 \mathrm{~nm}, 10-16 \mathrm{j} /$ $\mathrm{cm}^{2}$ ) were applied, in this order: three along the inferior orbital rim, with the device in a vertical position trying to cover all the area close to the eyewear's edge, one behind the lateral canthus, and one along the inferior orbital rim with the device placed horizontally.

After the IPL treatment, the protective eyewear was removed and the LLLT mask was applied. It contains a series of LEDs at wavelengths of $633 \pm 10 \mathrm{~nm}$, with an emission power of $103 \mathrm{~mW} / \mathrm{cm}^{2}$. During the 15 minutes of treatment, a total fluence of $110 \mathrm{j} / \mathrm{cm}^{2}$ is applied in the treated area. The periorbital area was treated with the patient keeping her/his eyes closed, to completely encompassing both lids. No manual gland expression was carried out after IPL/LLLT sessions. 


\section{Measures}

The following outcomes were assessed at baseline, posttreatment ( 3 months), and 6 and 12 months after the end of treatment: the OSDI, a 12-item self-reported scale widely used to assess dry eye symptoms (range 0-100, higher scores indicate more severity); Tear film osmolarity was measured with the TearLab ${ }^{\mathrm{TM}}$ Osmolarity System (TearLab Corporation, USA); non-invasive break-up time (NIBUT) and meniscus height were measured with the Keratograph $^{\circledR} 5 \mathrm{M}$ (OCULUS, Germany). For osmolarity, NIBUT and meniscus height, the average value of the two eyes was calculated for each patient and introduced in the analyses.

\section{Statistical Analysis}

Means and standard deviations of the dependent variables were calculated at baseline and for each follow-up. A repeated-measures analysis of variance (ANOVA) was carried out for each outcome, and paired $t$-tests were used to compare means at each time point, with Bonferroni's adjustment for multiple comparisons. For the OSDI, an exploratory interaction analysis was carried out using twoway repeated-measures ANOVA, with the conventional treatment (topical antibiotics, topical steroids, both treatments, none) as the between-subject factor, in order to assess whether the intervention effect differed between conventional treatments.

For the OSDI, the rate of patients with a score $\geq 33$ (severe symptoms) was calculated at 6 months prebaseline, baseline, post- and last follow up, and compared by means of $\chi^{2}$-test. Pearson's correlations among the four dependent variables were calculated. Finally, we performed two multiple linear regressions on the OSDI scores' change from pre- to post-intervention and 12month later, respectively, with age, sex, duration of DED, antibiotic and steroid treatment, and baseline OSDI, NIBUT, osmolarity and meniscus height (as well as their changes from baseline to each time point, in separate models) as independent variables.

\section{Results}

Two hundred and thirty-one patients underwent the procedure at the clinic, from which 156 met the inclusion criteria for analysis. Table 1 shows their sociodemographic and clinical characteristics. Mean age was $54.0(\mathrm{sd}=14.1$, range $30-77$ ) and $79.5 \%$ were women. Mean duration of
Table I Sociodemographic and Clinical Characteristics at Baseline

\begin{tabular}{|c|c|}
\hline & $n=156$ \\
\hline Age, (mean, sd, range) & $\begin{array}{c}54.0(14.1) \\
(30-77)\end{array}$ \\
\hline Female & 124 (79.5\%) \\
\hline Fitzpatick skin type & \\
\hline I & $3(1.9 \%)$ \\
\hline II & $4(2.6 \%)$ \\
\hline III & $48(30.8 \%)$ \\
\hline IV & $92(59.0 \%)$ \\
\hline V & $9(5.8 \%)$ \\
\hline Duration of DED in years (mean, sd, range) & $\begin{array}{c}5.9(3.6) \\
(1-12)\end{array}$ \\
\hline Conventional treatment during study: & \\
\hline - Artificial tears & $156(100 \%)$ \\
\hline - Topical antibiotics & 97 (62.2\%) \\
\hline - Topical steroids & $52(33.3)$ \\
\hline
\end{tabular}

Abbreviation: DED, dry eye disease.

DED was 5.9 years $(\mathrm{sd}=3.6)$ and mean OSDI score was $58.3(\mathrm{sd}=26.5)$.

There were no ocular or facial adverse effects in any patient during treatment or follow-up. Regarding effectiveness, Table 2 and Figures 1-4 show the results on the dependent variables at each time point. Repeatedmeasures ANOVA yielded significant results for the four variables ( $\mathrm{p}$-values $<0.001$ ). Between 6 months before and baseline, the three physiological measures (ie, osmolarity, NIBUT and meniscal height) significantly worsened ( $p$ values $<0.001$ ), whereas the change in the OSDI was not significant $(\mathrm{p}=1.000)$. At posttreatment, OSDI scores decreased by 44.0 points $(95 \%$ CI 38.1, 50.0; $<<0.001$ ), and increased again until the last follow up, but maintaining a significant reduction compared to baseline (MD $=-30.0,95 \%$ CI -23.4 , -36.8) (Table 2, Figure 1). The effect was not significantly different between groups of patients depending on their topical treatment (antibiotics, steroids, both or none) $(\mathrm{F}=1.39, \mathrm{p}=0.200)$.

Six months before baseline, 125 patients $(80.1 \%)$ had a score $\geq 33$ in the OSDI. This percentage was similar at baseline $(121,77.6 \%)$, reduced to $0 \%$ at post-treatment $\left(\chi^{2}\right.$ $=197.6, \mathrm{p}<0.001)$ and increased again to $39.1 \%$ at 12 months, but still significantly lower than the baseline rate $\left(\chi^{2}=84.2, \mathrm{p}<0.001\right)$. 


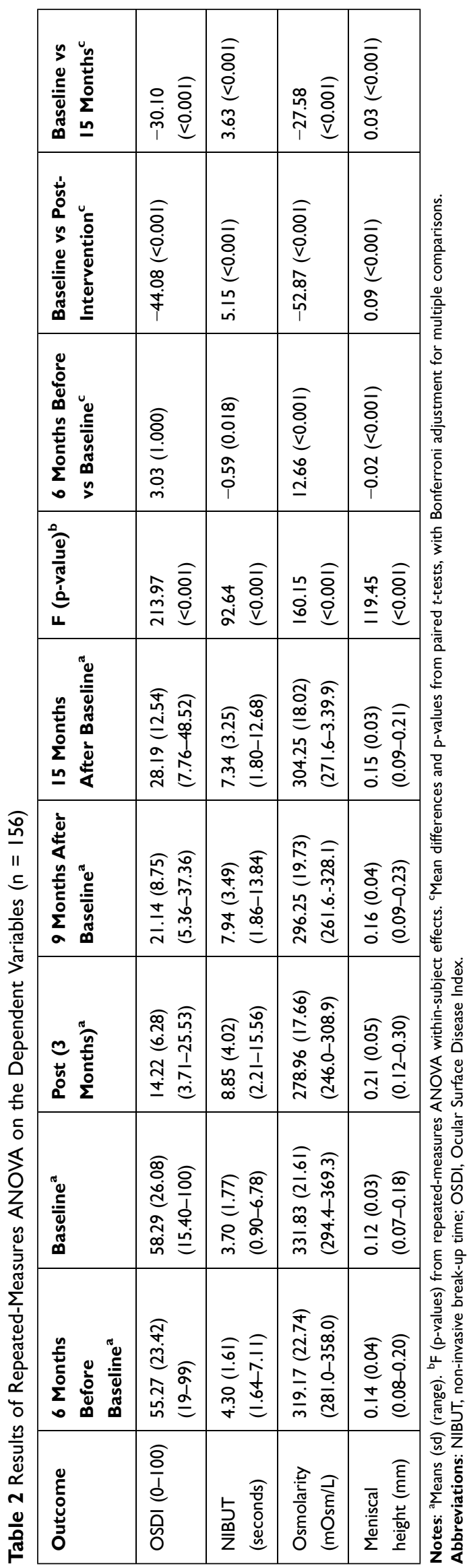

The other three variables showed the same pattern of change than the OSDI, improving significantly at postintervention and then deteriorating but maintaining significant clinical benefits 12 months after (all p-values $<0.001$, Table 2, Figures 2-4). Post-intervention and last follow-up improvements compared to baseline were, respectively, 5.2 (95\% CI 4.1, 6.1) and 3.6 seconds (95\% CI 2.8, 4.5) for the NIBUT; $53.0(95 \% \mathrm{CI} 46.8,59.0)$ and $28 \mathrm{mOsm} / \mathrm{L}$ (95\% CI 21.2, 34.0) for osmolarity, and 0.09 (95\% CI $0.08,0.10)$ and $0.03 \mathrm{~mm}(95 \%$ CI $0.02,0.04)$ for meniscus height.

There were no significant correlations between outcome variables at baseline or last follow-up (Table 3). At post treatment, OSDI significantly correlated with meniscal height $(\mathrm{r}=0.19, \mathrm{p}=0.02)$ and osmolarity with NIBUT $(\mathrm{r}=0.17, \mathrm{p}=0.03)$. Table 4 shows the multiple regression models predicting the change in the OSDI. Higher (worse) scores on the OSDI at baseline significantly predicted a greater scores' reduction at post $(\mathrm{B}=0.97, \mathrm{p}<0.001)$ and 12 months follow up ( $\mathrm{B}=1.02,95 \%$ CI 0.95, 1.10; $\mathrm{p}<0.001$ ). Older age was significantly related to a greater change at 12 months, although with a small effect $(\mathrm{B}=$ $-0.19, p=0.003)$. None of the other variables obtained significant results.

\section{Discussion}

The results of this study show an important reduction of symptoms and signs of DED after four sessions of IPL/ LLLT, in a sample with high symptoms' severity on average. Subsequently, outcomes worsened again but maintained significant benefits compared to baseline 12 months after the end of treatment. To our knowledge, the only published study that applied the combined IPL/LLLT is Stonecipher et al. ${ }^{18}$ This study observed a reduction in the rate of patients with severe symptoms (OSDI $\geq 33$ ) from $70.4 \%$ to $29.1 \%$, a nearly doubled TBUT and lower MGD grade between 1 and 3 months after one treatment session. Our results on the OSDI and NIBUT after the fourth session, 3 months after starting treatment, were better (no patients with severe symptoms and 139\% improvement in NIBUT), supporting the added benefit of successive sessions. Unfortunately, outcomes were not assessed between sessions in order to analyze the onset and evolution of improvement. Previous studies with IPL have shown immediate effects after the first session, and a peak of improvement between the last session (usually the third or fourth) and last follow-up, 1-2 months after. $^{11,12,22,23}$ Studies with longer follow-up have 


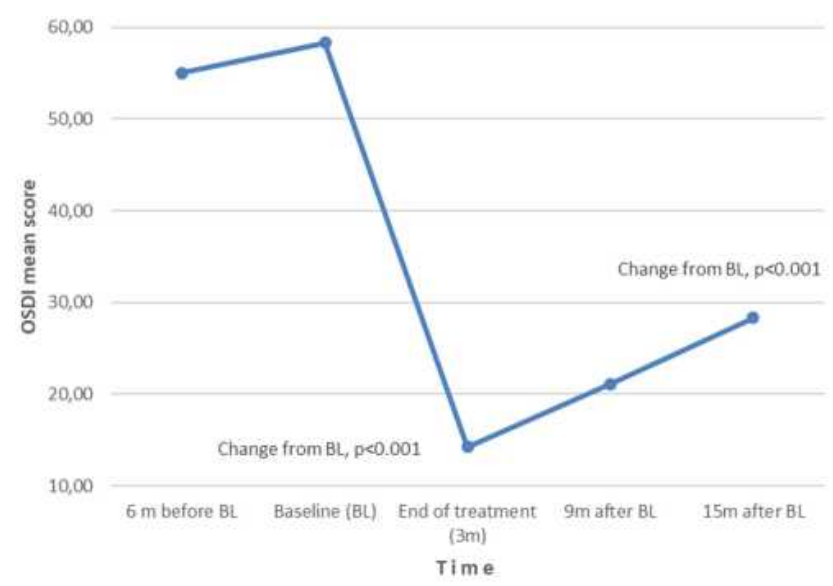

Figure I Change in OSDI scores.

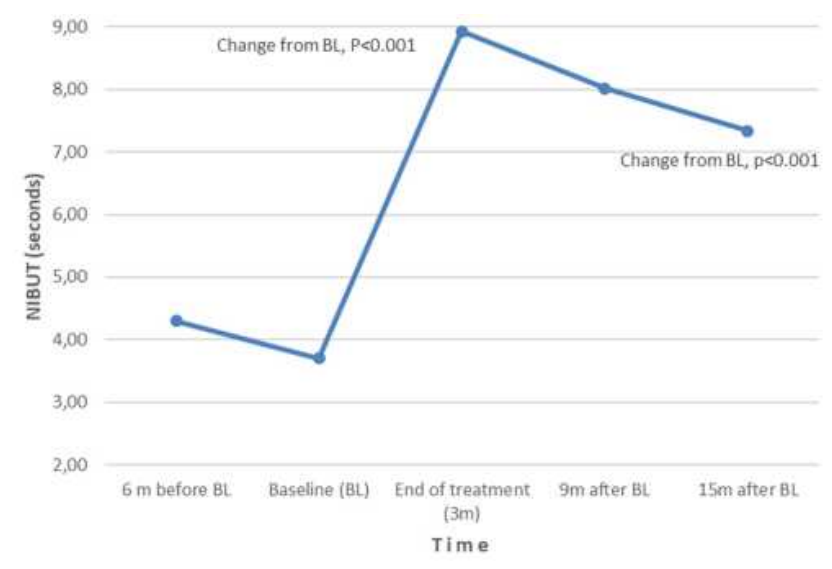

Figure 2 Change in NIBUT.

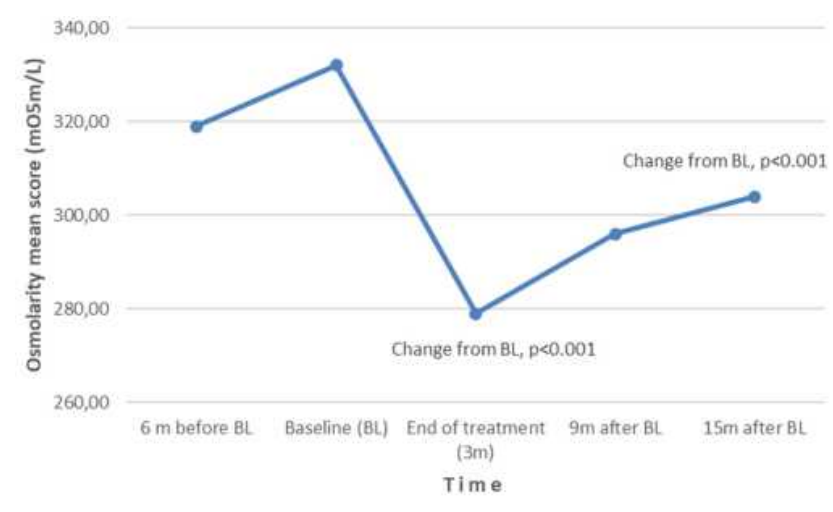

Figure 3 Change in osmolarity.

observed a decline of improvement 3-4 months after the last session, ${ }^{24-26}$ as occurred in our study at 6 months. More research is needed to determine the optimal number and timing of sessions, or even the possibility of applying individualized protocols based on patient's response.

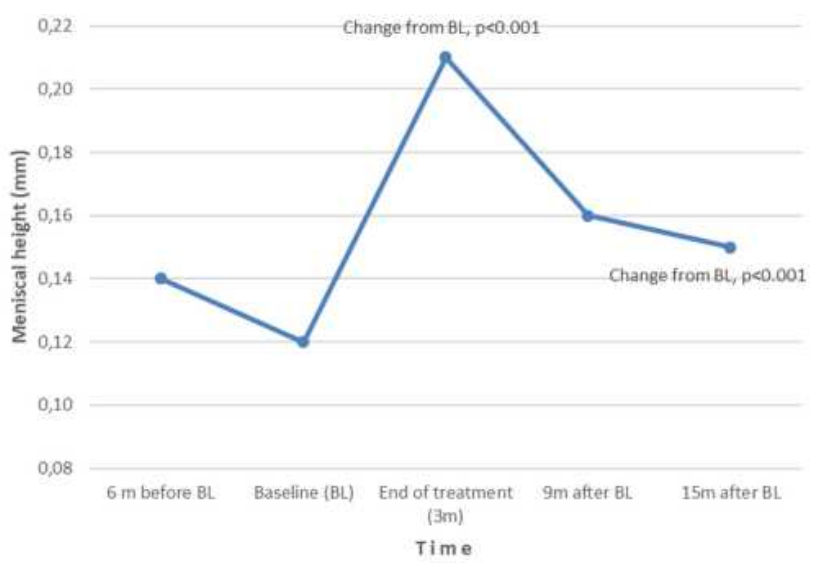

Figure 4 Change in meniscal height.

The study design does not allow to discriminate the differential effect of IPL and LLLT, a necessary aim since the effectiveness of the latter is currently supported by very limited evidence. We are only aware of two small studies that applied LLLT alone in patients with DED, obtaining significant improvements in the OSDI, ${ }^{17} \mathrm{TBUT}^{16,17}$ and $\mathrm{MGD}^{17}$ after 1-3 months. Regarding IPL, although there are several published case series, ${ }^{13}$ the strongest evidence on its efficacy comes from some sham-controlled ${ }^{10-12,24}$ and non-masked comparative ${ }^{14,27}$ randomized trials. Results have shown significant improvements in $\mathrm{TBUT}^{10-12,14,27}$ lipid layer thickness, ${ }^{10,14}$ lid margin parameters, ${ }^{14}$ MGD measures ${ }^{11,12,14,27}$ and symptoms, ${ }^{11,14,24}$ some of which were maintained until 4.5 and 7 months after the end of treatment. ${ }^{11,24}$ Comparing our results to the IPL withingroup change of these studies, our increase in breakup time $(139 \%)$ was lower than only two of them, which applied a similar IPL protocol to ours $\left(167 \%\right.$ in NIBUT $^{10}$ and $453 \%$ in fluorescein $\mathrm{TBUT}^{11}$ ). However, we obtained a significant reduction in tear film osmolarity not observed in these two studies. Furthermore, symptoms' reduction assessed by the OSDI was very intense in our study $(75.6 \%$ at post-intervention and $51.6 \%$ one year later), compared to the range observed in all the mentioned trials (16-33\%), except Arita et at ${ }^{14}$ with the Standardized Patient Evaluation of Eye Dryness (SPEED) questionnaire (reduction of $62 \%$ ), who applied 8 sessions of IPL plus Meibomian gland expression. Several confounders could be affecting these differences. Our sample had greater severity in osmolarity and symptoms at baseline, and therefore a greater potential for improvement; indeed, baseline scores in the OSDI significantly predicted posterior improvement. Conventional treatment was not interrupted during IPL/ 
Table 3 Pearson's Correlations Between the Dependent Variables $(n=156)$

\begin{tabular}{|l|c|c|c|c|c|c|c|c|c|}
\hline & \multicolumn{3}{|c|}{ Baseline } & \multicolumn{2}{c|}{ Post-Treatment (3 Month) } & \multicolumn{3}{c|}{ Last Follow Up (15 Months) } \\
\hline Outcome & NIBUT & Osmolarity & $\begin{array}{c}\text { Meniscal } \\
\text { Height }\end{array}$ & NIBUT & Osmolarity & $\begin{array}{c}\text { Meniscal } \\
\text { Height }\end{array}$ & $\begin{array}{c}\text { NIBUT } \\
\text { Osmolarity }\end{array} \begin{array}{c}\text { Meniscal } \\
\text { Height }\end{array}$ \\
\hline OSDI & -0.15 & 0.05 & 0.04 & 0.03 & 0.09 & $0.19 *$ & -0.02 & -0.04 & 0.09 \\
NIBUT & & 0.06 & -0.04 & & $0.17^{*}$ & 0.06 & & -0.13 & 0.08 \\
Osmolarity & & 0.09 & & & 0.13 & & -0.03 \\
\hline
\end{tabular}

Note: ${ }^{*} \mathrm{p}<0.05$.

Abbreviations: NIBUT, non-invasive break-up time; OSDI, Ocular Surface Disease Index.

Table 4 Multiple Linear Regression Models Predicting Change in the OSDI $(n=156)$

\begin{tabular}{|c|c|c|c|c|}
\hline \multirow[b]{2}{*}{ Age } & \multicolumn{2}{|c|}{ Change Baseline - Post Intervention ${ }^{\mathbf{a}}$} & \multicolumn{2}{|c|}{ Change Baseline - I5 Months ${ }^{a}$} \\
\hline & $-0.03(0.421)$ & $-0.03(0.403)$ & $-0.19(0.010)$ & $-0.19(0.010)$ \\
\hline Female & $1.51(0.231)$ & $1.70(0.171)$ & $2.41(0.334)$ & $2.29(0.361)$ \\
\hline Duration of DED & $0.08(0.585)$ & $0.02(0.88 I)$ & $0.15(0.594)$ & $0.19(0.504)$ \\
\hline Treated with antibiotics & $-1.08(0.306)$ & $-0.98(0.34 I)$ & $1.92(0.359)$ & $1.67(0.424)$ \\
\hline Treated with steroids & $0.63(0.568)$ & $0.65(0.552)$ & $1.49(0.493)$ & $1.56(0.478)$ \\
\hline Baseline OSDI & $-0.97(<0.001)$ & $-0.97(<0.001)$ & $-1.02(<0.001)$ & $-1.01(<0.001)$ \\
\hline Baseline NIBUT & $-0.14(0.637)$ & - & $-0.36(0.538)$ & \\
\hline Change in NIBUT & - & $0.03(0.810)$ & - & $-0.13(0.639)$ \\
\hline Baseline osmolarity & $-0.01(0.690)$ & - & $-0.02(0.700)$ & \\
\hline Change in osmolarity & - & $0.02(0.424)$ & - & $0.01(0.816)$ \\
\hline Baseline meniscal height & $-29.2(0.076)$ & - & $24.4(0.453)$ & \\
\hline Change in meniscal height & - & $21.1(0.211)$ & - & $7.64(0.718)$ \\
\hline
\end{tabular}

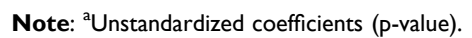

Abbreviations: DED, dry eye disease; NIBUT, non-invasive break-up time; OSDI, Ocular Surface Disease Index.

LLLT, although their interaction with the intervention was not significant.

In summary, with the current evidence, it is not possible to draw definitive conclusions about the added effect of LLLT on signs' and symptoms' improvement, but our results, in a sample with severe refractory symptoms and one of the longest published follow-up, justify more research. Contrary to IPL, the mechanism of action of LLLT is supposed to be athermal and related to cellular photoactivation, which may induce activation of mitochondria and antiinflammatory processes through the regulation of reactive oxygen species. ${ }^{15,28-30}$

DED is a heterogeneous disease and signs show a low correlation among them and with symptoms. ${ }^{31}$ Tear film osmolarity has been shown to be an acceptable marker of DED, ${ }^{32,33}$ but we have not found a significant correlation with symptoms, as occurred with the other outcomes.
This study has several limitations. First, a selection bias may be present since only patients with available measures were analyzed. Although our clinical experience shows that a great majority of treated patients were satisfied with the results of IPL/LLLT, we cannot discard this potential bias. Second, we did not assess the grade of MGD quantitatively, although the qualitative examination with meibography did not reveal clinically relevant changes in glands' macrostructure. However, this measure does not seem sensitive to IPL effects, since other studies that used meibography-based measures ${ }^{11,12,14,25,34,35}$ have also not found statistically or clinically significant differences, even when they did it with measures of meibum quality and expressibility, or lid margin parameters. Third, the study did not have a parallel control group with sham treatment, in order to control for placebo effects. Nonetheless, the intensity and duration of treatment effects, compared to those observed in previous IPL studies in eyes treated with sham treatment, ${ }^{10-12}$ suggest an actual clinically significant benefit. 


\section{Conclusions}

Light therapy is safe, and its application is easy and quick, thus representing a promising treatment option for a prevalent condition like DED. Our study supports the efficacy of IPL in the treatment of severe DED symptoms; however, more research is needed to define the number and timing of sessions that maximize the intensity and durability of its effects, what patients will benefit most, and the possibility of developing individualized protocols. In the case of LLLT clinical research is still incipient, and future studies should compare its efficacy against sham treatment, IPL and their combination. At the same time, advances in the knowledge of the biologic mechanisms responsible for the therapeutic effects of each technique should help to refine their indication, based on patients' characteristics. The present study, despite its limitations, offers valuable preliminary evidence on the long-term effectiveness of this combined therapy for the treatment of DED.

\section{Abbreviations}

ANOVA, analysis of variance; DED, dry eye disease; IPL, intense pulsed light; LED, light-emitting diodes; LLLT, low-level light therapy; MGD, Meibomian Gland Dysfunction; MD, mean difference; NIBUT, Noninvasive Breakup Time; OSDI, Ocular Surface Disease Index; SPEED, Standardized Patient Evaluation of Eye Dryness; TFOS-DEWS, Tear Film and Ocular Surface Society; TBUT, Tear film Breakup Time.

\section{Acknowledgments}

We thank Francisco García, Sílvia Rodriguez and Yurena Rodríguez for their work attending to patients and applying the evaluated treatment.

\section{Author Contributions}

All authors made a significant contribution to the work reported, whether that is in the conception, study design, execution, acquisition of data, analysis and interpretation, or in all these areas; took part in drafting, revising or critically reviewing the article; gave final approval for the version to be published; have agreed on the journal to which the article has been submitted; and agreed to be accountable for all aspects of the work.

\section{Funding}

This study has not received any kind of funding.

\section{Disclosure}

MA Pérez-Silguero declares that Topcon Corporation (Tokyo, Japan), distributor of the Eye-light ${ }^{\circledR}$ device, paid his travel and hotel costs for two national congresses organized by the Spanish Society of Ophthalmology (Granada, 26th-29th September 2018 and Madrid, 25th-28th September 2019), in which he directed two symposiums about dry eye. The remaining authors have no conflicts of interest.

\section{References}

1. Stapleton F, Alves M, Bunya VY, et al. TFOS DEWS II epidemiology report. Ocul Surf. 2017;15(3):334-365.

2. McDonald M, Patel DA, Keith MS, Snedecor SJ. Economic and humanistic burden of dry eye disease in Europe, north america, and Asia: a systematic literature review. Ocul Surf. 2016;14(2):144-167. doi:10.1016/j.jtos.2015.11.002

3. Chan TCY, Chow SSW, Wan KHN, Yuen HKL. Update on the association between dry eye disease and meibomian gland dysfunction. Hong Kong Med J. 2019;25(1):38-47. doi:10.12809/ hkmj187331

4. Jones L, Downie LE, Korb D, et al. TFOS DEWS II management and therapy report. Ocul Surf. 2017;15(3):575-628.

5. Toyos R, McGill W, Briscoe D. Intense pulsed light treatment for dry eye disease due to meibomian gland dysfunction; a 3-Year Retrospective Study. Photomed Laser Surg. 2015;33(1):41-46. doi:10.1089/pho.2014.3819

6. Raulin C, Greve B, Grema H. IPL technology: a review. Lasers Surg Med. 2003;32(2):78-87. doi:10.1002/1sm.10145

7. Liu R, Rong B, Tu P, et al. Analysis of cytokine levels in tears and clinical correlations after intense pulsed light treating meibomian gland dysfunction. Am J Ophthalmol. 2017;183:81-90. doi:10.1016/ j.ajo.2017.08.021

8. Ahmed S, Taher IE, Ghoneim D, Safwat AM. Effect of intense pulsed light therapy on tear proteins and lipids in meibomian gland dysfunction. J Ophthalmic Vis Res. 2019;14(1):3-10. doi:10.4103/ jovr.jovr_12_18

9. Choi M, Han SJ, Ji YW, et al. Meibum expressibility improvement as a therapeutic target of intense pulsed light treatment in meibomian gland dysfunction and its association with tear inflammatory cytokines. Sci Rep. 2019;9(1):1-8. doi:10.1038/s41598-018-37186-2

10. Craig JP, Chen Y-H, Turnbull PRK. Prospective trial of intense pulsed light for the treatment of meibomian gland dysfunction. Investig Opthalmology Vis Sci. 2015;56(3):1965-1970. doi:10.1167/ iovs.14-15764

11. Piyacomn Y, Kasetsuwan N, Reinprayoon U, Satitpitakul V, Tesapirat L. Efficacy and safety of intense pulsed light in patients with meibomian gland dysfunction - a randomized, double-masked, sham-controlled clinical trial. Cornea. 2019;39(3):325-332. doi:10.1097/ICO.0000000000002204

12. Rong B, Tang Y, Tu P, et al. Intense pulsed light applied directly on eyelids combined with meibomian gland expression to treat meibomian gland dysfunction. Photomed Laser Surg. 2018;36(6):326-332. doi:10.1089/pho.2017.4402

13. Giannaccare G, Taroni L, Senni C, Scorcia V. Intense pulsed light therapy in the treatment of meibomian gland dysfunction: current perspectives. Clin Optom. 2019;11:113-126. doi:10.2147/OPTO. S217639

14. Arita R, Fukuoka S, Morishige N. Therapeutic efficacy of intense pulsed light in patients with refractory meibomian gland dysfunction. Ocul Surf. 2019;17(1):104-110. doi:10.1016/j.jtos.2018.11.004 
15. Kim W-S, Calderhead RG. Is light-emitting diode phototherapy (LED-LLLT) really effective? Laser Ther. 2011;20(3):205-215. doi:10.5978/islsm.20.205

16. Toyos R, Briscoe D, Toyos M. The effects of red light technology on dry eye disease due to meibomian gland dysfunction. JOJ Ophthalmol. 2017;3(5):3-5.

17. Stonecipher K, Komm C, Potvin R. Low level light therapy as an adjunct treatment for meibomian gland dysfunction. Acta Sci Ophthalmol. 2020;3(11):13-18. doi:10.31080/ASOP.2020.03.0177

18. Stonecipher K, Abell TG, Chotiner B, Chotiner E, Potvin R. Combined low level light therapy and intense pulsed light therapy for the treatment of meibomian gland dysfunction. Clin Ophthalmol. 2019;13:993-999. doi:10.2147/OPTH.S213664

19. Craig JP, Nichols KK, Akpek EK, et al. TFOS DEWS II definition and classification report. Ocul Surf. 2017;15(3):276-283. doi:10.1016/j.jtos.2017.05.008

20. Schiffman RM. Reliability and validity of the ocular surface disease index. Arch Ophthalmol. 2000;118(5):615-621. doi:10.1001/ archopht.118.5.615

21. Fitzpatrick TB. The validity and practicality of sun-reactive skin types I through VI. Arch Dermatol. 1988;124(6):869-871. doi:10.1001/archderm.1988.01670060015008

22. Dell SJ, Gaster RN, Barbarino SC, Cunningham DN. Prospective evaluation of intense pulsed light and meibomian gland expression efficacy on relieving signs and symptoms of dry eye disease due to meibomian gland dysfunction. Clin Ophthalmol. 2017;11:817-827. doi:10.2147/OPTH.S130706

23. Albietz JM, Schmid KL. Intense pulsed light treatment and meibomian gland expression for moderate to advanced meibomian gland dysfunction. Clin Exp Optom. 2018;101(1):23-33. doi:10.1111/ cxo. 12541

24. Rong B, Tang Y, Liu R, et al. Long-term effects of intense pulsed light combined with meibomian gland expression in the treatment of meibomian gland dysfunction. Photomed Laser Surg. 2018;36 (10):562-567. doi:10.1089/pho.2018.4499

25. Seo KY, Kang SM, Ha DY, Chin HS, Jung JW. Long-term effects of intense pulsed light treatment on the ocular surface in patients with rosacea-associated meibomian gland dysfunction. Cont Lens Anterior Eye. 2018;41(5):430-435. doi:10.1016/j.clae.2018.06.002
26. Karaca EE, Evren Kemer Ö, Özek D. Intense regulated pulse light for the meibomian gland dysfunction. Eur J Ophthalmol. 2018;30 (2):289-292. doi:10.1177/1120672118817687

27. Gao YF, Liu RJ, Li YX, et al. Comparison of anti-inflammatory effects of intense pulsed light with tobramycin/dexamethasone plus warm compress on dry eye associated meibomian gland dysfunction. Int $J$ Ophthalmol. 2019;12(11):1708-1713. doi:10.18240/ ijo.2019.11.07

28. Sommer AP. Mitochondrial cytochrome c oxidase is not the primary acceptor for near infrared light - it is mitochondrial bound water: the principles of low-level light therapy. Ann Transl Med. 2019;7(S1): S13-S13. doi:10.21037/atm.2019.01.43

29. Walski T, Dąbrowska K, Drohomirecka A, et al. The effect of red-tonear-infrared (R/NIR) irradiation on inflammatory processes. Int $J$ Radiat Biol. 2019;95(9):1326-1336. doi:10.1080/ 09553002.2019.1625464

30. George S, Hamblin MR, Abrahamse H. Effect of red light and near infrared laser on the generation of reactive oxygen species in primary dermal fibroblasts. J Photochem Photobiol B Biol. 2018;188:60-68. doi:10.1016/j.jphotobiol.2018.09.004

31. Bron AJ, Tomlinson A, Foulks GN, et al. Rethinking dry eye disease: a perspective on clinical implications. Ocul Surf. 2014;12(2):S1-31.

32. Versura P, Profazio V, Campos EC. Performance of tear osmolarity compared to previous diagnostic tests for dry eye diseases. Curr Eye Res. 2010;35(7):553-564. doi:10.3109/02713683.2010.484557

33. Potvin R, Makari S, Rapuano C. Tear film osmolarity and dry eye disease: a review of the literature. Clin Ophthalmol. 2015;9:2039-2047. doi:10.2147/OPTH.S95242

34. Arita R, Mizoguchi T, Fukuoka S, Morishige N. Multicenter study of intense pulsed light therapy for patients with refractory meibomian gland dysfunction. Cornea. 2018;37(12):1566-1571. doi:10.1097/ ICO.0000000000001687

35. Yin Y, Liu N, Gong L, Song N. Changes in the meibomian gland after exposure to intense pulsed light in meibomian gland dysfunction (MGD) patients. Curr Eye Res. 2018;43(3):308-313. doi:10.1080/ 02713683.2017 .1406525
Clinical Ophthalmology

\section{Publish your work in this journal}

Clinical Ophthalmology is an international, peer-reviewed journal covering all subspecialties within ophthalmology. Key topics include: Optometry; Visual science; Pharmacology and drug therapy in eye diseases; Basic Sciences; Primary and Secondary eye care; Patient Safety and Quality of Care Improvements. This journal is indexed on PubMed

\section{Dovepress}

Central and CAS, and is the official journal of The Society of Clinical Ophthalmology (SCO). The manuscript management system is completely online and includes a very quick and fair peer-review system, which is all easy to use. Visit http://www.dovepress.com/ testimonials.php to read real quotes from published authors. 